\title{
Deep Convolutional Neural Network Approach as a Universal Tool for Determination of Local 3D Structure from ABF STEM Images of Perovskites
}

\author{
Nouamane Laanait ${ }^{1}$ and Albina Y. Borisevich ${ }^{2}$ \\ 1. Computational Sciences \& Engineering Division, Oak Ridge National Laboratory, Oak Ridge, TN. \\ 2. Materials Science and Technology Division, Oak Ridge National Laboratory, Oak Ridge, TN.
}

Among the multiple deep learning approaches available today, systems geared towards the analysis of imaging data, mostly convolutional neural networks and variants thereof, are of particular interest for microscopy data interpretation. The ability of these deep artificial neural networks to capture the salient and abstract features present in images and to use such features to perform specific tasks is an especially attractive aspect for e.g. automated determination of local structural distortions in perovskites from atomic resolution data.

Scanning transmission electron microscopy (STEM) via the annular bright field mode (ABF) can be optimized for imaging light element columns such as oxygen, and has been extensively used for structural studies of tilted perovskites, in particular at heterointerfaces where structure can change on a unit-cell scale. Intriguingly, recent studies demonstrated that shapes of oxygen columns in ABF images contain information on local 3D tilt structure beyond the apparent projection geometry of the image; qualitative symmetry information, and, in a small subset of cases, quantitative structural information can be retrieved [1]. However, automating this analysis with a simple filter/transform workflow appears problematic: signal from the cation columns provides most of the image contrast, yet most of the structural information is concentrated in comparatively subtle oxygen atomic columns, necessitating considerable human input to isolate oxygen column signal prior to quantification. We have therefore decided to explore if deep learning can be harnessed for this purpose.

Due to very large training sets $(\sim 100,000$ and above) required for training of a deep convolutional neural network (DCNN), as well as very uneven distribution of real-world compounds among theoretically possible structure types, we used dynamical electron scattering simulations for one composition $\left(\mathrm{SrTiO}_{3}\right)$ to generate the needed training sets. The DCNN was then trained on most of the generated data, while a small subset was reserved for validation. Validation studies have demonstrated that DCNN approach can extract both symmetry and magnitudes of octahedral rotations with remarkable accuracy across the entire span of structure types. DCNN trained on simulated data was also able to analyze experimental images. Fig.1 shows experimental ABF STEM micrograph of a uniform region of a $\mathrm{CaTiO}_{3}$ thin film and the corresponding maps of the tilt angles $\alpha, \beta$, and $\gamma$; while there are some local variations in parameters, no features are apparent. In contrast, similar image of a $\mathrm{CaTiO}_{3} / \mathrm{LSAT}$ interface region shows large variation at the interface for $\alpha$ and $\beta$, with impact in the film as well as in the substrate (Fig. 2). DCNN approach was also successful in determination of tilt parameters from the images of materials classes it did not see during training, such as $\mathrm{GdFeO}_{3}$-type, highlighting the ability of the method to focus on only the relevant features [2]. Data on incorporation of sample mistilt and residual aberrations into DCNN training regiment will also be presented [3]. 
References:

[1] Qian He et al, ACS Nano 9 (2015), p. 8412.

[2] N. Laanait, Q. He, A. Borisevich, to be submitted (2018).

[3] This work used resources of the Oak Ridge Leadership Computing Facility at Oak Ridge National Laboratory, which is supported by the Office of Science of the Department of Energy under Contract DE-AC05-00OR22725. NL acknowledges support from ORNL's Laboratory Director's R\&D fund. AYB acknowledges support from DMSE, DOE BES.

(a)

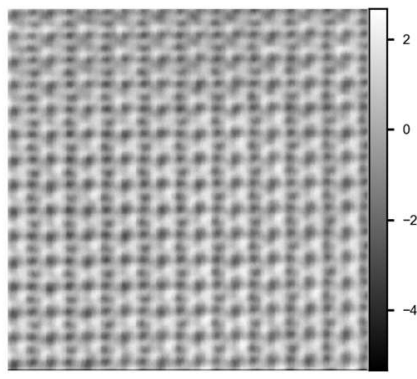

(b)

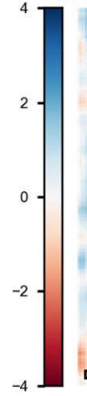

(c)

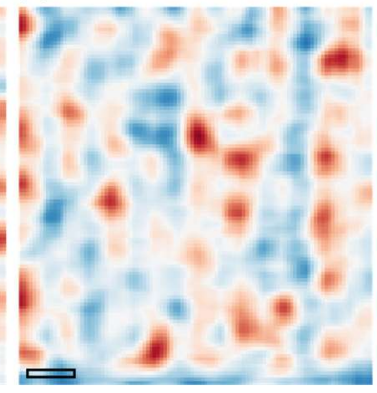

(d)

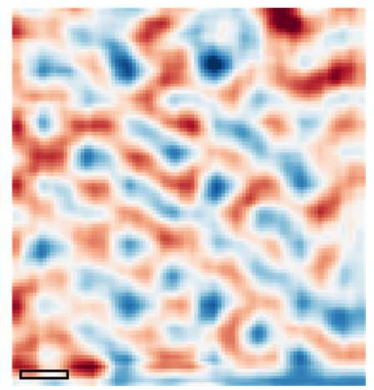

Figure 1. (a) ABF STEM image in (110)pc projection of a uniform region of $\mathrm{CaTiO}_{3}$ thin film, (b)-(d) local octahedral tilt angles $\alpha, \beta$, and $\gamma$ determined from (a) using DCNN approach. Scale bar is one unit cell $(0.4 \mathrm{~nm})$

(a)

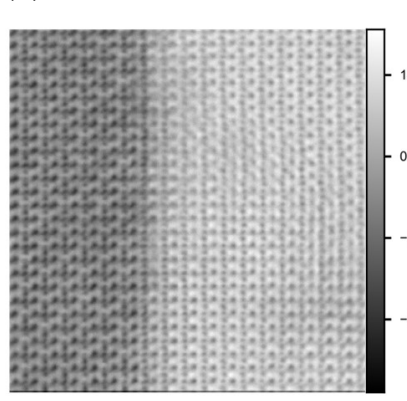

(b)

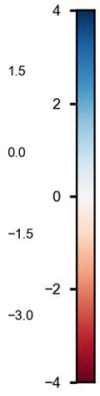

(c)

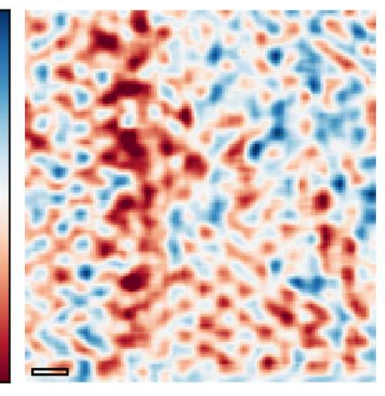

(c)

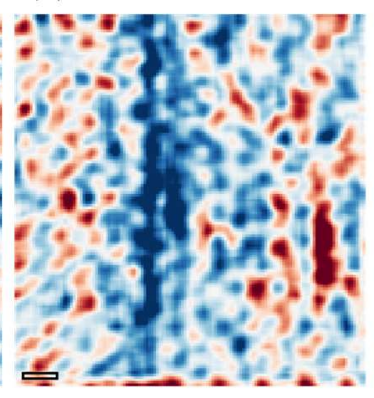

(d)

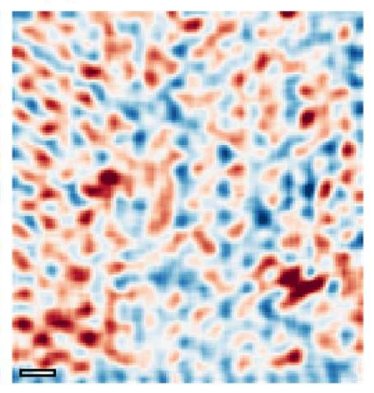

Figure 2. (a) ABF STEM image in (110) pc projection of a $\mathrm{CaTiO}_{3} / \mathrm{LSAT}$ interface, (b)-(d) local octahedral tilt angles $\alpha, \beta$, and $\gamma$ determined from (a) using DCNN approach. Scale bar is one unit cell (0.4 $\mathrm{nm})$. 